\title{
Robotic-assisted laparoscopy for the excision of a pelvic leiomyosarcoma
}

\author{
Grant Chavin - Matthew Gettman
}

Received: 8 October 2007 / Accepted: 5 December 2007 / Published online: 8 January 2008

(C) Springer London 2008

\begin{abstract}
The indications for robot-assisted laparoscopic surgery in Urology are expanding as surgical experience with robotic surgery mounts. We describe our experience of performing a laparoscopic robot-assisted excision of a pelvic leiomyosarcoma in an adult.
\end{abstract}

Keywords Leiomyosarcoma $\cdot$ Robot-assisted laparoscopy

\section{Introduction}

Robot-assisted laparoscopic surgery is being used increasingly to address pathology within the pelvis. Urologists, gynecologists, and general surgeons are rapidly expanding the indications for this procedure. Notable applications include robot-assisted laparoscopic hysterectomy, myomectomy, tubal reanastomosis, sacrocolpopexy, and cancer staging [1]. A number of studies have confirmed improved or equivalent patient outcomes in general surgical procedures, including robot-assisted laparoscopic cholecystectomy, Nissen fundoplication, Heller myotomy, gastric banding procedures, and Roux-en-Y gastric bypass [2]. Improved three-dimensional imaging, magnification, and improved dexterity of the robotic system have contributed to its growing popularity.

\section{G. Chavin · M. Gettman ( $\bowtie)$}

Departments of Urology, Mayo Clinic,

200 First Street SW, Rochester, MN 55905, USA

e-mail: gettman.matthew@mayo.edu

G. Chavin

e-mail: chavin.grant@mayo.edu
Robotics is currently being applied to address both upper tract and lower tract pathology, such as nephrectomy, partial nephrectomy, pyeloplasty, ureteral reimplantation and sacrocolpopexy. Expanding urologic application has been bolstered in large part by the success that robot-assisted laparoscopic surgery has seen with the treatment of clinically localized prostate cancer. Robotic prostatectomy is being performed at large academic centers and in the community with surgical results approaching those of open radical retropubic prostatectomy [3-5]. We report a case of robot-assisted laparoscopic excision of a pelvic leiomyosarcoma in an adult.

\section{Case report}

A 47-year-old man with a past medical history significant for MEN1 syndrome was referred to Urology in 2004 for an incidentally found indeterminate pelvic mass on CT scan. The patient originally underwent partial pancreatectomy approximately 15 years prior to presentation for multiple insulinomas. The patient subsequently had a recurrence of insulinomas in the head of the pancreas and underwent enucleation of these lesions. His postoperative course was complicated by a duodenal ulcer for which the patient presented with nausea, vomiting, and anemia. A CT scan of the abdomen and pelvis was performed to fully evaluate these clinical symptoms. A $3-\mathrm{cm}$ indeterminate solid mass was seen on this study at the left posterolateral aspect of the bladder. A follow-up MRI was ordered to further characterize this mass (Fig. 1). MRI of the pelvis without and with IV contrast demonstrates a well-circumscribed ovoid lesion measuring $3 \mathrm{~cm}$ insinuated between the bladder and left seminal vesicle. It appeared to be extrinsic to the bladder causing an impression on the posterior bladder wall and 


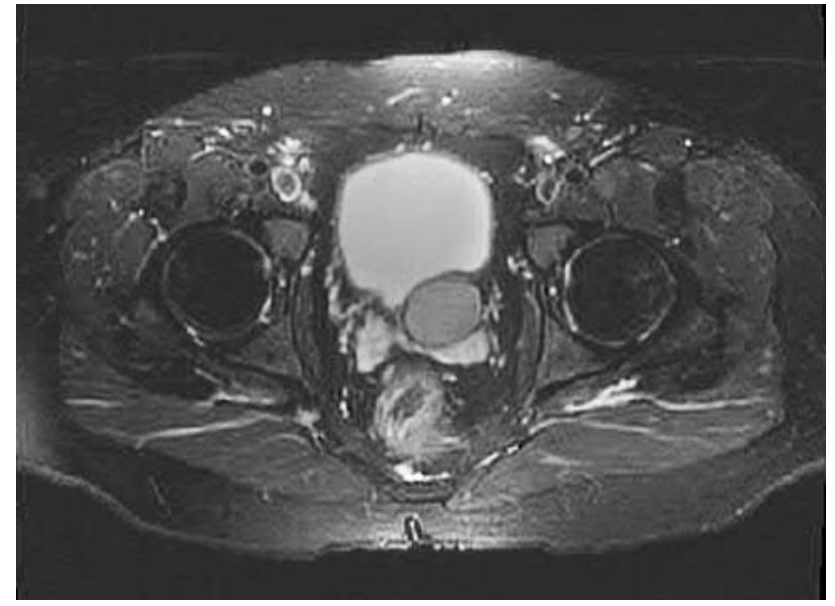

Fig. 1 MRI cross-sectional image of the pelvic mass posterior located in the retrovesical cul de sac

compressing the seminal vesicle. There did appear to be a fat plane separating the mass from the bladder. On post-gadolinium images it demonstrated mild diffuse enhancement consistent with a solid mass, but was otherwise indeterminate.

After giving informed consent, the patient underwent laparoscopic robot-assisted excision of his pelvic mass. Pneumoperitoneum was established, and the first trocar was placed $2 \mathrm{~cm}$ above the umbilicus in the midline. The laparoscope was now positioned, and we identified significant adhesions from the patient's prior abdominal surgery. After lysis of adhesions, remaining trocars were placed in a fanlike configuration on the abdomen as used for robotassisted radical prostatectomy. A three-arm da Vinci robotic system was installed. The pouch of Douglas was now identified. Initial dissection was performed using the ProGrasp on the left robotic arm and the hook electrocautery on the right robotic arm. A $30^{\circ}$ downward lens was used for the entire dissection. Using electrocautery, we incised into the pouch of Douglas and were able to immediately identify the vas deferens. The vas was followed down toward the ampulla of the vas to the level of the seminal vesicle. The hook electrocautery was now exchanged for round tip scissors. The seminal vesicle was identified and carefully dissected with limited cautery. As needed, the hook electrocautery was exchanged with the scissors to provide point electrocautery. Limited cautery was used to prevent injury to the pelvic plexus. With the seminal vesicle freed, the mass was noted to be sitting above our dissection. Using sharp and blunt dissection, we were able to dissect this circumferentially. There was a pedicle arising in the inferior medial aspect which was clipped, and the mass was then placed into an entrapment sack. This mass was intimately associated with the ureter and was compressing not only the seminal vesicle but also the bladder. After the lesion had been removed, the ureter was unharmed as well as the vas deferens, seminal vesicles and bladder. There was excellent hemostasis at the level of the surgical bed.

Pathologic review demonstrated a $3.4 \times 2.6 \times 2.6 \mathrm{~cm}$ soft tissue mass consistent with low grade leiomyosarcoma. Surgical margins were negative. Postoperatively the patient was evaluated by medical oncology and radiation oncology, however adjuvant therapy was not recommended. At 8-month follow-up the patient had no evidence of recurrent disease based on interval cross-section imaging.

\section{Discussion}

Urologic sarcomas, in general, are very rare and account for only $2 \%$ of all soft tissue sarcomas. In order of frequency, these tumors are found in the paratesticular tissues, kidney, prostate and bladder [7]. Leiomyosarcoma and liposarcoma are the two most common histologic subtypes. Most patients who undergo complete surgical excision for primary disease will have prolonged disease specific survival. Conversely, positive surgical margin status confirms an increased risk of both metastatic disease and death from disease [7].

Histology represents another important prognostic variable. Low-grade sarcomas have favorable long-term outcome compared with high grade sarcomas. Specifically, these sarcomas are associated with an actuarial 5-year-survival of $82-100 \%$ [8, 10]. Radical open surgical excision with negative surgical margins has traditionally been the treatment of choice for localized sarcomas with adjuvant radiotherapy in selected patients [10].

Open surgical dissection in the region of the seminal vesicles, while providing excellent results, can be associated with significant morbidity. Specifically, bladder wall injury, rectal injury, ureteral injury, and injury to the neurovascular bundle can be associated with this dissection. Laparoscopic surgery on normal seminal vesicles in patients with prostate cancer was first described by Kavoussi et al. [13]. He demonstrated that the transperitoneal approach both suitable access and excellent visualization of the retrovesical seminal vesicles. This allows for identification and control of the blood supply and careful dissection of the seminal vesicles away from the bladder and prostate. Seminal vesical cysts may also be approached in this manner. We employed this strategy using robot-assisted laparoscopy to identify and excise a retrovesical mass.

Robot-assisted laparoscopic surgery represents an important technical development in the Urologists' therapeutic arsenal. Robotic surgery is a minimally invasive approach that continues to grow in popularity. Frequently promoted advantages of this procedure include less intraoperative blood loss, better visualization, shorter hospital stay, 
and more rapid patient recovery. For these reasons, the procedure has a broad and expanding application in urologic surgery. Robotic surgery has been used with success in the management of localized cancer of the prostate, bladder cancer, renal surgery and adrenal surgery. Oncologic outcomes are the true test of the applicability of robot-assisted laparoscopy in Urology. Preliminary results from large series of radical prostatectomies are promising and compare favorably to the open surgical approach $[3,4]$.

In this report, we describe the feasibility of retrovesical dissection of the seminal vesicles and excision of a pelvic leiomyosarcoma using robot-assisted laparoscopy. In comparison to standard laparoscopy, we found that the robotic approach improved maneuverability. The three-dimensional imaging system also was beneficial in understanding the relationship of the mass to the ureter, bladder, and seminal vesicle. In addition, the articulated instruments improved the precision of the dissection. Additional advances in robotic technology and instrumentation since this case was performed appear to further increase the feasibility of the approach, yet this remains to be formally proven. From a standpoint of instrumentation, introduction of bipolar cautery, endoshears, and retraction devices since 2004 would likely increase the ability to resect pelvic tumors. In addition, use of the new robotic systems with a fourth arm and increased maneuverability (such as the daVinciS robotic system) would also likely be of importance when operating in the pelvis. Nonetheless, we show that when using even the most basic of robotic instrumentation that this approach is a reasonable and safe alternative to open surgery or pure laparoscopic surgery for access to the retrovesical cul de sac.

\section{References}

1. Advincula AP, Song A (2007) The role of robotic surgery in gynecology. Curr Opin Obstet Gynecol 4:331-336

2. Hazey JW, Melvin WS (2004) Robot-assisted general surgery. Semin Laparosc Surg 2:107-112

3. Menon M, Shrivastava A, Kaul S, Badani KK, Fumo M, Bhandari M, Peabody JO (2007) Vattikuti institute prostatectomy: contemporary technique and analysis of results. Eur Urol 3:648-657

4. Patel VR, Thaly R, Shah K (2007) Robotic radical prostatectomy: outcomes of 500 cases. BJU Int 5:1109-1112

5. Patel VR, Tully AS, Holmes R, Lindsay J (2005) Robotic radical prostatectomy in the community setting-the learning curve and beyond: initial 200 cases. J Urol 1:269-272

7. Dotan ZA, Tal R, Golijanin D, Snyder ME, Antonescu C, Brennan MF, Russo P (2006) Adult genitourinary sarcoma: the 25-year Memorial Sloan-Kettering experience. J Urol 176(5):2033-2038 discussion 2038-2039

8. Froehner M, Lossnitzer A, Manseck A, Koch R, Noack B, Wirth MP (2000) Favorable long-term outcome in adult genitourinary low-grade sarcoma. Urology 56(3):373-377

10.Russo P, Brady MS, Conlon K, Hajdu SI, Fair WR, Herr HW et al (1992) Adult urological sarcoma. J Urol 147:1032

13.Kavoussi LR, Schuessler WW, Vancaillie TG, Clayman RV (1993) Laparoscopic approach to the seminal vesicles. J Urol 150(2 Pt 1):417-419 